\title{
XLIII.
}

\section{Ueber den Humus und die Wirkung des Düngers bei der Ernährung der Pflanzen.}

\author{
Von
}

\section{z. Soubeiran.}

(Journ. de Pharm. et de Chim. XVII, 321.)

Die Centralackerbaugesellschaft des Departements der unteren Seine stellte die Fragen:

„Sind die Kohlensäure, die Luft, das Wasser, das Ammoniak und die unorganischen oder mineralischen Substanzen die einzigen Körper die zur Entwickelung der Pflanzen beitragen?:"

"Ueben die organischen Bestandtheile der Dammerde und des Düngers nur dadurch einen günstigen Einluss auf die Pllanzen aus, dass sie durch ihre freiwillige und langsame Zersetzung Kohlensäure und Ammoniak liefern?"*

„Es ist die Aufgabe gestellt, die chemische Geschichte des Humus oder der Dammerde zu erōrtern und besonders zu prüfen, ob die Substanz, die man Ulminsäure nennt, wesentlich bei dem Ernährungsakte der Pflanzen mitwirkt. Es ist genaı die Rolle des Humus zu bestimmen und der Antheil der mineralischen und der organischen Substanzen zu untersuchen, die sich in dem Humus tinden."

„Es ist endlich auf experimentellen Wege zu untersuchen, ob; wie Theodor von Saussure, den Ansichten mebrerer Chemiker und Physiologen zuwider, annimmt, das lösliche Extrakt der Dammerde unverändert mit Wasser in die absorbirenden Gefässe der Pflanze übergeht."

Die Gesellschaft lenkt die Aufmerksamkeit der Beobachter auf die Wirkungr des Humus bei der Veretation. Dieser Punkt der Wissenschaft schien auf positive Weise festgestellt zu sein, als er von den Chemikern in Frage gestellt wurde. Es ist nothwendig, diese Frage unverzüglich zu entscheiden, damit man entweder dem Humus die Hauptrolle, die man demselben bis jetzt in der Düngertheorie gab, wiederertheile, oder demselben seinen in der neuern Zeit bestimmten untergeordneten Platz 
anweise. Die Gesellschaft wünschte eine schnelle Antwort und hat zu diesem Behufe den 1. Juli als Termin für die Ablieferung der Abhandlungen bestimmt. Die Versuche der Concurrenten wurden in einem Zeitraume beendigt, der nicht eine Periode der Vegetation umfasst. Ich glaube aber, dass die in gegenwärtiger Abhandlung niedergelegten Thatsachen hinreichend sein werden, um die Haupterscheinung bei der Ernährung durch den Humus aufzuklären. Später werde ich dieselben durch noch nicht beendigte Versuche vervollständigen, die aber das bis jetzt beobachtete nur bestätigen kōnnen.

In der ersten Abtheilung meiner Abhandlung werde ich die chemische Natur des Humus erürtern und die Rolle desselben bei der Ernährung der Pflanzen aufzuklären suchen. In der zweiten Abtheilung werde ich einige Düngerarten mit Rücksicht auf den Humus und die Substanzen, welche denselben bilden können, prüfen. $Z u$ gleicher Zeit werde ich die Gelegenheit wahrnehmen, einige Irthümer aufzuklïren, die Methoden der Analysen der Düngerarten verbessern und hauptsächlich einige Axiome, die in der Wissenschaft als absolutes Gesetz dastehn, auf ihren wahren Werth zurückführen.

\section{Erste Abtheilung.}

\section{Ueber den Eumus}

In der ersten Abtheilung dieser Abhandlung will ich die Eigenschaften des Humus, den Zustand, in welchem er sich in der Dammerde befindet, und seinen Einfluss auf die Vegetation festzustellen suchen. Es sind die Fragen zu lōsen, ob der Humus den Pflanzen als direktes Nahrungsmittel dient, oder ob derselbe nur durch die bei seiner Gährung entwickelte Kohlensăure wirkt, oder ob er ein Rückstand, ein nutzloses Caput mortuum ist. Ich studirte zuerst die bisher veröffentlichten Arbeiten und bemerkte, dass die Widersprüche und die augenscheinlich irrigen Ansichten mich nüthigen mussten, mich nur auf meine eigenen Versuche zu verlassen. Glücklicher Weise gelang es mir, einige Wahrheiten festzustellen und mebrere Irrthümer zu beseitigen.

Hinsichtlich des Düngers sind die Beobachtungen der prak- 
tischen Landwirthe in Allgemeinen wenig beweisend und widersprechen sich oft. Die chemische Zusammensetzung der nüngemittel und die Beschaffenheit des Bodens ist ihnen oft nicht hinlänglich bekannt. Daraus folgt, dass die erhaltenen Resultate, so getreu wie sie unter den gegebenen Umständen auch sind, nicht generalisirt werden können, ohne dass man bei jedem Schritte auf Unzulässigkeiten träfe.

Den Chemikern wirft man andererseits nicht mit Unrecht vor, dass sie auf eine kleine Anzahl von Thatschen gestützt, sich zu leicht theoretischen Ansichten hingeben. Diejenigen, die sich vertrauensvoll diesen Ansichten lingeben, sind nur zu oft getãuscht worden. Die Rahhschläge der Münner der Wisscnschaft sind dadurch in einen oft nur zu sehr gegründeten Mlislredit gekommen, welcher der Einführung der wissenschaflichen Erfahrung in die Praxis die grōssten Schwierigkeiten entgegenstellt.

Es sind besonders drei Theorien über die Düngerarten herrscliend. Diese Theorien enthalten aber die Wahrheit obgleich sie anscheinend im Widerspruch stehn. Eine jelle derselben ist ein Fortschritt, unter der Bedingmg, dass man keiner cinen absoluten Werth zuschreibt und die beiden anderen ausschliesst.

Die Landwirthe behaupten, dass die humusreichsten Ackerboden die besten seien, dass der Dünger die Bestimmung habe, dem Boden Humus zu geben, und dass der letztere für die Pflanzen das vorzüglichste Nahrungsmittel sei. Fin Dünger ohne Humus ist folglich nach dieser Ansicht ein unwirksames Ding. Liebig nimnt hingegell an, dass der llumus nur ein unwesentlicher Nebenbestandtheil des Düngers sei. Dieser Chemiker sagt, dass man einer Pflanze nur die zu ihrer Existenz nothwendigen mineralischen Bestandtheile zu geben brauche, damit dieselben verhältnissmässig die bluterzengenden Principe bilden, nach welchen sich der Werth unserer Nahrungsmittel richtet. Nach Liebig ist der Humus niemals direktes Nahrungsmittel für die Pflanze; er trāgt nur indirekt zur Eruährung durch die bei seiner Zersetzung entstehende Kohlensäure bei. Die französische Schule endlich, an deren Splitze Boussingault und Payen stehen, nimmt an, dass der Stickstoffichalt den Massstab für den Werth der Nahrungsmittel abgäbe. Beide Ge- 
lehrte gingen sogar so weit, nach ilrrem Princip eine Aequivalententafel aufzustellen.

Die Aufgabe erscheint nach dem Yorstehenden für jeden, der sich mit systematischen Ideen nicht befasst, vollständig gelöst. Die Landwirthe schreiben mit Recht der Gegenwart des Humus in Dünger eine grosse Wirksamkeit zu. Liebig war ebenfalls vollkommen im Rechte, als er den Einfluss der Salze als Reizmittel auf die Vegetation und als wesentlich constituirende Elemente einiger Nabrungsstoffe hervorhob. Boussingault und Payen hatten endlich ibre gewichtigen Gründe, als sie angaben, dass der Werth eines Düngemittels mit seinem Gehalte an Stickstoff zunehme. Derjenige hat aber sicher das grösste Recht auf seiner Scite, der da anuimmt, dass der beste Dünger der sei, der zu gleicher Zeit die drei Hauptbestandtheile: Humus, Salze und stichstoffhaltige Substanz enthält.

Der Humus komint aus der Dammerde und rührt von der langsamen Verresung der Holzfaser her. Bei Gegenwart von Luft und von Feuchtigkeit verbrennt ein Theil des Wasserstoffs der Holzfaser und so wie das Gleichgewicht einmal gestort ist, bildet sich Fohlensüure auf Kosten der zurückbleibenden Elemente. Das Resultat dieser Einwirkung ist eine allmählige Zunabme in der Menge des Kohlenstonis, die indess ihre Grenze hat, ua die Verwandschaft des Wasserstoffs zum Kohlenstoff in dem Masse der Verwesung hindernd entgegentritt, als das Ilolz armer an Wasserstofl wird. Diese Verwandschaft hält endlich der oxydirenden Einwirkung der Luft das Gleichgewicht.

Der Humas, die schwarse Dammerde (terreau charbonneux) und der Hoder sind die drei Glieder, die man bei der allmähligen Zersetzung des Holzes wahrgenommen zu haben glaubt. Der Humus charakterisirt sich durch seine Lòslichkeit in den Alkalion; die schuar $\approx e$ Dammerde ist darin unlöslich; an der Lult aber giebt sie Kohlensäure aus und wird dadurch löslich: Der Boder ist nach Liebig das Endprodukt der Zersetzung des Holzes. Ebenso wie das Holz enthält der Moder Samerstoff und Wasserstoff in dem Verhältniss, um Wasser zu bildell, nur ist der Koblenstoffgehalt weit beträchtlicher. Nach Li ebigs ist derselbe nur Lei Gegenwart von Alkalien făhig, auf den Sauerstoff der Luft zu wirken, und ein ilem Humus ähnliches, $\cdot$ losliches Produkt zu erzeigen. 
Die Reihe dieser Verbindungen ist nicht gehorrig festgestellt; das erste Glied derselben ist jedenfalls die Substanz, dit man mit dem Namen der schwarzen Dammerde bezeichnet hat; ich behalte diese Benennung nur deshalb bei, weil es unnöthig ist, einen neuen Namen für ein Uebergangsprodukt zu erfinden, das jeden Augenblick seine Zusammensetzung verändert. Die schwarze Dammerde entsteht durch die Einwirkung der Luft auf das Holz, ehe sich durch diese Ein๘irkung der Humus bildet. Bei fortgesetzter Einwirkung entsteht der Humus; daher kommt es auch, dass erschopfte Dammerde beim Liegen an der Luft nach einer längeren Zeit von Neuem löslichen Humus giebt.

Der Humus ist, wie schon erwähnt, der in alkalischen Losungen lősliche Theil der Dammerde. Was den Hoder anbelangt, so ist dessen Existenz hypothetisch. Kein Versuch hat bis jetzt die Existenz eines Korpers in der Dammerde nachgewiesen, der kohlenstoffreicher als der IIumus wäre, und die merkwürdige Eigenschaft bätte, die Reihe der Zersetzungsprodulite wieder hinaufzusteigen und unter Mitwirkung der Alkalien und der Luft einen Körper zu liefern, der weniger Kohlenstolf enthält als er selbst *).

Ich stellte meine Versuche mit einer Dammerde an, die mir ron Herrn Neumann, Obergärtner des Jardin des plantes übergeben worden war. Dieselbe war im dritten Jahre und eine von denjenigen Sorten, die von den Gemüsegärtnern nicht gesucht sind, weil sie nicht hitzig sind, obgleich dieselben eine üppige Vegetation entwickein.

Alkohol zog daraus nur einige unwesentliche fette oder harzähnliche Substanzen aus. Wasser färble sich gelb und ers. nach sehr langer Zeit entstand eine gefärbte Lossung. Dieselbe enthielt eine kleine Menge von salpeter- und schwefelsauren Salzen, Chlormetallen und Spuren von phosphorsauren Salzen. Diese Salze kōnnen wohl die Ursache der Aufoustung der organischen Substanz sein.

*) Ich spreche hier nicht von dem Moder, der unter Wasser bei fehlendem Sauerstoffe entsteht. Ich land denselben in einer Dammerde, die sich am Fusse eines Baumes gebildet hatte. Er findet sich ferner im Miste, wenn die Luft nicht bis in das Innere der Haufen gelangen konnte. 
Verdünntes oder concentrirtes Ammoniak giebt bei Abschluss der Luft mit der Dammerde zusammengebracht, eine nur schwach gefärbte Flüssigkeit, in welcher Süuren einen geringen Niederschlag hervorbrachten. Bei Zutritt der Luft erzeugte sich aber, besonders unter Mitwirkung der Wärme eine braun gefärbte Lösung, in der Säuren einen starken Niederschlag erzeugten.

Dieser Versuch beweist, dass in der Dammerde wenig freier IIumus enthalten ist. Derselbe zeigt zugleich die reichliche Bildung dieses Körpers unter dem gleichzeitigen Einflusse der Luft und der Alkalien.

Eine grössere Henge Humus findet sich in der Dammerde an Kalk gebunden. Nan beweist diess, wenn man die Dammerde nit einer verdünnten Säure behandelt, auswächt und sodann mit ammoniakalischem Wasser behandelt. In diesem Falle erhält man direkt ohne Mitwirkungr der Luft eine sehr dunkle Lösung, in welcher Säuren einen starken Niederschlag erzeugen. Der entscheidende Versuch war aber folgender. Ich stellte humussauren Kalk durch Fällen einer ammoniakalischen Humuslösung mit Chlorcalcium dar. Der hunussaure Kalk wurde nach dem Auswaschen mit Aetzammoniak behandelt. In der Kälte sowoh! als in der Würme, nahm die Flüssigkeit kaum cine gelbliche Färbung an. Daraus folgt, dass die Färbung des Ammoniaks, die unmittelbar bei seiner Berülırung mit der Dammerde eintritt, durch die Auflösung des freien Ilumus, nicht aber durch Entziehung des Humus aus dem humussauren Kalke entsteht.

Erselzt man bei diesem Versuche das Ammoniak durch lohlensaures Ammonials, so erhält man sowohl mit reinem humussauren Kalk, als auch mit Dammerde dunkelgefürbte Flüssigkeiten. Dieser Versuch zeigt uns die Rolle des kohlensauren Ammoniaks im Dünger, die darin besteht, den an Kalk gebundenen Humus lōslich zll machen.

Die mit Wasser, Chlorwasserstoffsäure und Ammoniak erschŏpfte Dammerle erhält, wenn man sie der Luft aussetzt, die Eigenschaft, von Neuem mit Ammoniak eine gefürbte Flüssigkeit zu geben. Bei Gegenwart eines Alkalis geht die Umwandelung noch weit schneller vor sich. Als ich in einer Glocke über Quecksilber operirte, wies ich direkt die Absorption des Sauerstoffs der Luft und die Bildung von in Ammoniak löslichem Humus nach. Bei einem anderen Versuche, nahm die erschöpfte 
Dammerde an der Luft unter der Einwirkung des Ammoniaks schon nach einigen Augenblicken die Eigenschaft an, Wasser braun zu färben. Durch Waschen und Wiederholen des Versuchs konnte ich dasselbe Resultat viele Male hervorbringen. Die Bildung des Humus unter dem Einflusse der Alkalieh ist demnach unbestreitbar; die koblensauren Alkalien und besonders die ätzenden besitzen diese Eigenschaft in einem noch höheren Grade als das Ammoniak.

Es ist die Identität des vermittelst Alkalien ausgezogenen Humus mit dem in der Dammerde vorkommenden in Zweifel gezogen worden. Man hat jedoch durchaus keinen Grund, einen Unterschied zwischen diesen beiden Kürpern anzunehmen, und diess zwar um so weniger als der Humus niemals direkt, sondern erst, nachdem derselbe durch die Alkalien löslich geworden ist, von der Pflanze aufgenommen wird. Der Humus, welcher als Nahrungsmittel dient, war demnach demselben Einflusse ausgesetzt, als wenn derselbe direkt aus Ammoniak und Dammerde dargestellt worden wäre.

Ele ich weiter gehe will ich einige Worte über das sogenannte Humusextrakt (extrait de terreau) anführen. Einige verstehen unter dieser Bezeichnung denjenigen Theil des Humus, der sich beim Behandeln der Dammerle mit Wasser löst. In der That ist diess niclıts als Humus, dessen Lösung durch Kalkoder Alkalisalze begünstigt worden ist. Andere verstehen unter Humusextrakt den in Alkohol lōslichen Theil, den man erhält, wenn man, nachdem eine alkalische Humuslösung gefällt worden ist, den Niederschlag mit Alkohol behandelt. In diesem Falle ist es nicht der Humus, der sich lüst, sonder'n eine Verbindung von Humus mit der Säure, die zum Fällen angewendet worden ist. Ich fand, dass.Flumus, der vermittelst Schwefelsäure gefällt worden war, sich in Allsolol in reichlicher Menge lüste. Eine Lösung von Humus in einem Alkali wird bekanntlich durch Essigsäure nicht gefällt. Ich fällte Humus vermittelst Chlorwasserstoffsäure, wusch den Niederschlag längere Zeit hindurch mit destillirten Wasser; die ablaufende Flüssigkeit wurde kaum durch salpetersaures Silberoxyd getrübt. $\mathrm{Zu}$ diesem Zeitpunkte behandelte ich den Niederschlag sowohl in der Kälte als auch in der Wärme mit Alkohol. Darau prüfte ich die alkoholische Flüssigkeit und den unlöslichen Rückstand auf Chlor. Ich verfuhr dabei auf die 
Weise, dass ich die Flüssigkeit und den Rückstand trocknete und darauf mit kohlensaurem Natron glühte. Die Flüssigkeit gab einen sehr chlorhaltigen Rückstand. Der mit Alkohol erschōpfte Humus enthielt kein Chlor.

Die Zusammensetzung des Humus lässt sich nicht mit absoluter Genauigkeit bestimmen. Wenn man die ammoniakalische Lösung mittelst einer Säure fällt, so kann der niederfallende Humus mit gewissen Stoffen, die ihn schon in der Dammerde begleiten und ebenso wie der Humus die Eigenschaft haben, durch Alkalien gelōst und durch Säuren gefällt zu werden, verunreinigt sein, der Versuch kann uns aber über die Zusanamensetzung des Humus aufklären. Nachdem dic Dammerde mit verdünnter Chlorwasserstoffsüure und Wasser gewaschen worden war, behandelte ich dieselbe mit Ammoniak und fällte den $\mathrm{Hu}$ mus durch Chlorwasserstoffsãure. Nachdem ich denselben durch Waschen mit destillirtem Wasser gereinigt hatte, zog ich den Humus mit Alkohol und mit Aether aus.

Polydor B oullay fand in künstlichem Ulmin:

\section{6,92 Kohlenstoff \\ 43,08 Wasser.}

Péligot nimmt an, dass das Ulmin 72,3 p. C. Kohlenstoff enthalte. Er glanbt, dass Boullay den Kohlenstoff nur unvollständig verbrannt habe; es lässt sich aber nicht annehmen, dass dieser geschickte Chemiker einen Irrthum von 15 p. G. hinsichtlich des Wassergehaltes hätte begehen können. Ich werde bald erklāren, auf welche Weise beide Chemiker so verschiedene Resultate erhalten konnten.

Die Analyse des aus der Dammerde dargestellten Ulmins giebt keine constanten Zahlen. Stets findet sich darin Stickstoff, das Verhältniss der anderen Elemente ist veränderlich. Die Nichlübereinstimmung von Analysen, deren Genauigkeit nicht in Zweifel gezogen werden konnte, liess mich endlich erkennen, dass die Zusammensetzung des aus der Dammerde ausgezogenen Humus veränderlich ist und dass der Kohlenstoff darin um so mehr vorwaltet, je länger derselbe seit seiner Bildung der Luft ausgesetzt gewesen war. Ich fand $52-56$ p. C. Kohlenstoff, nie mehr als 57 p. C. Es scheint als ob unter denjenigen Bedingungen der Bildung, bei welchen das Alkali verdünnt und die Temperatur eine mässige ist, der von Peligot bei dem 
künstlichen Ulmin gefundene Kohlenstoffgehalt nieht erreicht werden kann.

Ich überzéugte mich, dass das Verhältniss des liohlenstoffs in der That unter der fortgesetzten Einwirkung der Luft und eines Alkalis zunimmt. Ich löste in einer verdünnten Lösung von kohlensaurem Natron Humus auf, der 53 p. C. Kohlenstofr enthielt. Ich liess die Lösung vierzig Stunden lang sieden und fällte den Humus von Neuem; diessmal enthielt derselbe $57 \mathrm{p}$. C. Kohlenstoff. Der in Alkalien lösliche Humus zeigt also eine veränderliche Zusammensetzung. Es giebt einen Zeitpunkt, in welchem das Holz in löslichen Humus umgewandelt worden ist; von diesem Zeitpunkte an, nimmt das Verhültniss des Kohlenstoffs zu, ohne dass der entstandene Körper die Eigenschaften verliert, die ihn zur Ernährung der Pllanzen geeignet machen. Für den Humus giebt es keine genal bestimmten Verhäitnisse, ebenso wenig wie für alle organischen Körper, die durch allmähliche Einwirkung entstehen, es giebt da eine Reihe von Uebergängen, zwischen denen sich lieine Grenze feststellen lässt.

Ich halte es für unnöthig, die für die verschiedenen IItmusvarietäten gefundenen Zahlen anzuführen. Eine derselben, mit einer scliwachen Aetznatronlösung dargestellt, gab mir bis zu 56,4 p. C. Kohlenstoff, Der Stickstoff macht stets einen Bestandtheil des Humus aus; die Menge desselben variirt zwischen $\mathbf{2 - 2 , 5}$ p. C. Ich führe nun die Besultate an, die ich mit Humus, der aus altem Holze ausgezogen worden war, erhalten habe.

Man trifft bekanntlich in den Wäldern oft alte Bäume,. deren Stamm sich im Innern allmählich zersetzt und sich endlich in ein mehr oder minder dunkel gefärbtes Pulver verwandelt. Bei weit vorgeschrittener Zersetzung ist ein etwas starker Stoss hinreichend, um dieses Zersetzungsprodukt in grosser Menge zu erhalten. Das zu meinen Versuchen dienende zersetzte Holz stammte von einer Eiche aus dem Walde von Fontainebleau. Es war feucht, von gelbbrauner Farbe und batte alle Eigenschaften der reinsten Dammerde.

Die Substanz war ferner ohne Geruch und Geschmack, färbte Wasser nicht, gab aber mit Ammoniak eine sehr dunkle Lơsung; darauf zuerst mit einer Säure, dann abermals mit Ammoniak behandelt, färble sich letzteres von Neuem. Das auf 
diese Weise ausgezogene Holz gab endlich unter Mitwirkung der Luft und der Alkalien von Neuem eine gefärbte Flüssigkeit. Das Pulver des alten Holzes bestand demnach aus einem Gemenge von reinem Humus, etwas humussaurem Kalk und einer noch nicht ungewandelten Substanz, die aber bei Luftzutritt unter dem Einfluss der Alkalien in Humus überzugehen fähỉg ist.

Ich zog den Humus aus altem Holz aus, indem ich dasselbe mit Wasser und alsdann mit Ammoniak wusch. Diese Lüsung wurde vermittelst Chlorwasserstoffsäure gefallt und der Humus durch Waschen mit Wasser, siedendem Alkohol und Aether gereinigt. Der so dargestellte Humus wurde analysirt. Er hinterliess 7,16 p. C. Asche. Nach der Methode von Will und Varrentrapp erhielt ich 2,5 p. C. Stickstoff.

Die Verbrennung mit Kupferoxyd und cllorsaurem Kali gab, nach Abzug der Asche, folgende Resultate:

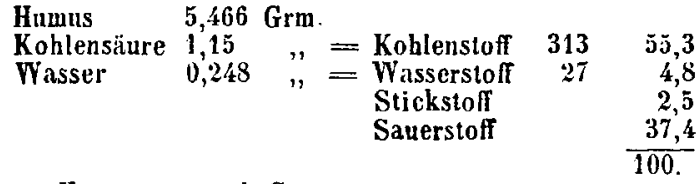

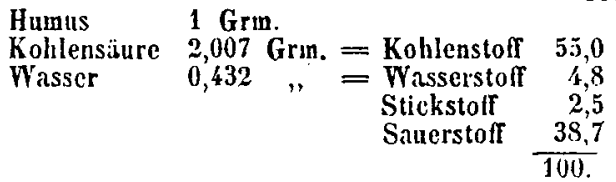

Lüsst man bei diesen Analysen den Stickstoff unberücksichtigt, so führen diese Zahlen zu der Formel:

$$
\mathrm{C}_{34} \mathrm{H}_{28} \mathrm{O}_{18}
$$

Es ist besonder's hervorzuheben, dass in diesem Humus, der durch jahrelange Einwirkung von Luft und von Feuchtigkeit entstanden ist, der Kohlenstoffgehalt $\mathbf{5 5}$ p. C. nicht überschritt. Es scheint, als ob diese Menge die ausserste Grenze sei, die durch die Zersetzung des Holzes, wenn man Hitze und concentrirte Alkalien nicht anwendet, erreicht werden kann. Diese Grenze ist wie man sieht weit von der Kohlenstoffmenge (72 p. C.) entfernt, die Péligot in dem kunnstichen Ulmin gefunden hat.

Obgleich der Stickstoff stets einen Bestandtheil des Humus ausmacht, so lässt sich doch nicht angeben, welcher Theil zu den beigemengten stickstoffhaltigen Substanzen gehört. $E s$ ist bemerkenswerth, dass in dem Pulver von altem Eichenholz der 
Stickstoffgehalt grösser als in dem Eichenholz ist, aus welchem das erstere entstanden. Es ist daher wahrscheinlich, dass ein Theil des Stickstoffs der Luft während der Zersetzung fixirt worden ist; so ist wenigstens die Ansicht von The od or von S a u ssure. Man kŏnnte vielleicht annehmen, dass die Ueberreste von Insekten Ursache des bōberen Stickstoffgehaltes wären; seit langer Zeit aber konnte dieses konsistenzlose Pulver den Insekten nicht mehr zum Asyl dienen, da es durch die geringste Erschütterung auseinander fiel.

Liebig nimmt an, dass bei weiter vorgeschrittener Zersetzung sich der Humus in Moder verwandelt, der sich von anderen ähnlichen Zersetzungsprodukten durch einen grüsseren Kohlenstuffyehalt und ferner dadurch unterscheidet, dass die Luft nur bei Gegenwart von Alkalien auf ihn einzuwirken vermag. Es ist mir kein Versuch bekannt, der zu Annahme der Existenz dieşes Körpers Veranlassung geben kōnnte; seine Bildung ist durch die angefübrten Thatsachen sogar unwahrscheinlich geworden. Wir sehen in der That, dass reiner Humus, d. h. solcher, der durch Alkali gelöst und aus seiner Lósung durch eine Säure gefällt worden ist, auf die Luft fast ohne Einwirkung ist; nachdem derselbe sechs Monate lang unter Quecksilber aufbewahrt worden war, hatte er kaum das Volumen der Luft verăndert. Auf Zusatz von Ammoniak ist die Einwirkung markirter, immer aber noch eine sehr langsame, und es müssen lange Jahre dazu nothwendiog sein, ehe der Humus das Verhältniss von 72 p. Ct. Kohlenstoff erreichen kann, wobei derselbe in Ammoniak noch lóslich ist. Diese Grenze ist selbst in einem Pulver von altem Holz noch nicht erreicht, das vielleicht fünfzehn bis zwanzig Jahre lang der oxydirenden Luft ausgesetzt gewesen war.

\section{Die Einwirkung des Humus auf die Vegetation.}

Die Landwirthe glauben, dass der Humus den Pflanzen direkt zur Nahrung dient. Nach Liebig besteht sein Nutzen darin, dass derselbe, als in einer fortwährenden Zersetzung begriffen Kohlensäure bildet, die bestândig von den Wurzeln absorbirt wird. Mir scheint es, als ob Liebig die Dienste des Humus als Nahrungsmittel der Pflanzen zu gering angeschlagen hätte. Die Idee bekämpfend, dass die Dammerde in Gestalt von 
humussaurem Kalk von den Ptlanzen al iorbirt werden kơnne, sucht Liebig nach der Quantität der in. der Asche der Pflanzen gefundenen Basen und nach der Zusammensetzung der humussauren Salze festzustellen, dass die Kohlenstoffmenge, die auf diese Weise in die Pflanzen übergehe, nur ein sehr geringer Theil von der Totalmenge des Kohlenstoffs in den Pflanzen sei. Die geringe Lŏslichkeit des humussauren Kalkes liefert ihm ein neues Argument für seine Bebauptung. Beide Ansichten verlieren aber ihre Bedeutung, weil ich gezeigt habe, dass der Humus in Form von humussaurem Ammoniak von der Pflanze aufgenommen wird. Das bei der Fäulniss des Düngers entstehende kohlensaure dmmoniak lóst den gebildeten Humus auf, und befördert dessen Bildung unter Mitwirkung der atmosphärischen Luft; dieses Ammoniak macht ferner den in Form von humussaurem Kalk in dem Boden entbaltenen Humus lơslich. Die Menge des von der Pflanze absorbirten Humus kann demnach weder durch die Löslichkeit des humussauren Kalkes im Wasser, noch durch die Aschenmenge der Pflanze bestimint werden. Das Ammoniak, welches als Auflosungsmittel gedient hat, wird in dem Pflanzengewebe umgewandelt und trägt direkt zur Bildung der stickstoffhaltigen Substanzen bei.

L i ebig hebt ferner hervor, dass die Wälder und die Wiesen die Qualitat des Bodens verbessern, ohne dass derselbe gedüngt wirl, obghteich man jedes Jahr Holz schlägt und Heu erntet. Die Pflanzen geben demnach dem Boden mehr als sie von demselben aufnehmen, und der bei der Ernte entzogene Kohlenstoff rührt allein aus der Atmosphäre her. Es ist diess kaum ein Beweis gegen den Nutzen des Humus, denn wenn der absorbirte Humus die Wirkung hat, den Pflanzen Nahrung zuzuführen, welche die Lebenskraft der Pflanze erhüht und dic Anzahl und das Volumen der Absorptionsorgane vermehrt, so muss die Pflanze desto reichlichere Nahrung aus der Atmosphäre aufnehmen. Der Humus ist demnach, ohne selbst Kohlenstoff abgegeben zu haben, das primum movens der reichlichen Produktion von Holz und andern Pflanzenkürpern. Es ist auserdem bekannt, dass in einem Boden, in welchem der Humus fehlt, die Vegetation stets spärlich und wenig ergiebig ist.

Nach den Ansichten von Liebig, nach welchen die Rolle der Dammerde sich darauf heschränkt, den Wurzeln die bei 
ihrer Zersetzung gebildete Kohlensäure zu liefern, hört der Humus auf, die Ursache dieser Kohlensãurebildung zu sein. Nicht der Humus zersetzt sich, sondern die Holzfaser, die schrvarze Dammerde. Sind beide Körper einmal in Humus verwandelt, so hören dieselben auf, eine Wirkung auszuüben, da der Humus zwar in Alkalien löslich ist, sich aber mit grosser Hartnäckigkeit bei Zutritt der Luft und bei Feuchtigkeit unverändert erhält. Durch Alkalien wird die Zersetzung kaum beschleunigt. $\mathrm{Zu}$ welchem Zwecke erzeugt die Natur einen unthätigen Körper? Keineswegs Jässt sich annehmen, dass dieser durch Alkalien und besonders durch kohlensaures Ammoniak löslich gemachte Körper, nicht von den Wurzeln absorbirt werde und zur Nahrung der Pflanze diene. Der Humus wirl aber absorbirt, wie aus folgenden Versuchen hervorgehen wird.

Ich nahm vorsichtig aus der Erde cinen ziemlich starken Stock von Lapsana communis, wusch die Wurzeln mit Wasser und tauchte dieselben in eine sehr verdünnte Lüsung von humussaurem Ammoniak, das durch langes Aussetzen in die atmosphärische Luft von allem überschüssigen Alkali befreit war. Die Flüssigkeit und die Wurzeln waren vor dem $Z$ utritt des Lichtes geschützt. Während der acht Tage, während welcher Zeit ich den Versuch fortsetzte, vegetirte die Pflanze üppig fort. Jeden Tag brachte ich die Wurzeln in cine neue Lösung, jeden Tag gab ich der Flüssigkeit, die Tags zuvor angewendet worden war, vermittelst destillirten Wassers ihr ursprüngliches Volumen. Die schwāctere Nüance der Flüssigkeit zeigte deutlich, dass ein Theil humussaures Ammoniak absorbirt worden war.

Im Jahre 1844 sãete ich in eine Erde, die durch Glühen von allen organischen Substanzen befreit worden war, zu welcher ich aber etwas Knochenerde und schwefelsaures hali gesetzt hatte, Hafer und Bohnen. Als die Pflanzen aufgegangen waren, benetzte ich sie jeden Tag mit einer schwachen Lösung von vollkommen neutralem humussauren Ammonit. Die Vegetation ging normal vor sich und ich erbielt Blüthen und Früchte. Es lässt sich durchaus nicht annehmen, dass bei diesen Versuchen das humussaure Ammoniak in Kohlensäure verwandelt worden wäre; die in der Lósung enthaltene Substanz ging vielmehr direkt in die Pllanze über, und gab den Stoff zur Nahrung derselben her. Das kräftige Gedeihen des Stockes der Lapsana, das Aufschos- 
sen des Hafers und der Bohnen, die bei beiden erhaltenen Blüthen und Früchte, sprechen deutlich für die günstigen Bedingungen, unter welchen sie sich entwickelten.

Es bleibt nur noch zu ergründen übrig, was aus dem Humus entsteht, sobald er von den Wurzelfasern aufyenommen worden ist, ob er darin sogleich eine Umwandlung erleidet oder ob derselbe weiter in den Saftgefässen fortgeführt wird. Die Beantwortung dieser Frage, über welche ich heute nichts anzutühren weiss, behalte ich mir für die Zukunft vor.

Die Rolle des Humus beschränkt sich nicht darauf, ein Nahrungsmittel für die Pllanzen abzugeben. Diese Substanz wirkt auch hygroskopisch, absorbirt die Feuchtigkeit der Atmosphäre und erhält dadurch die Erde frisch und feucht. Sie condensirt ferner das Ammoniak der Luft und wirkt in so fern fäulnisswidrig, als sie die Zersetzung der stickstoffhaltigen Substanzen mässigt, so dass die Pflanze ein allerdings nicht reichliches aber stets erneuertes Nahrungsmittel erhălt. Hätte auch der Humus selbst keine direkt näbrende Kraft, so sichern doch die angeführten schätzbaren Eigenschaften derjenigen Düngerarten, welche Humus mit andern nährenden Bestandtheilen enthalten, ihnen eine unbestreitbare Superiorităt zu. Zu bewundern ist das Walten der Natur, die durch den geheimnissvollen Prozess der Fäulniss, welche die Ueberreste todter Pflanzen vernichtet, Elemente zu einer reichlichen und nothwendigen Nahrung nachkommender Gencrationen schafft.

Aus den in vorstehender Abhandlung niedergelegten Thatsachen ziche ich folgende Schlüsse: Bei der Bildung der Dammerde ist die schwarze Dammerde (le terrau charbonneux) das erste Produkt; dasselbe unterscheidet sich von dem Holze durch einen grössern Kohlenstoffgehalt und von dem Humus durch einen geringern Gehalt desselben Elementes.

Das zweite Produkt dieser Zersetzung ist der Humus, der durch seine Loslichkeit in Ammonik charakterisirt ist.

In der gewŏhnlichen Dammerde ist ein Theil des Humus frei, ein bei weitem grōsserer aber an Kalk gebunden, vorhanden. Bei altem vermoderten Eichenholz findet das Umgekehrte statt. Der Humus ist stets stickstoffhaltig. Das Verhältniss des Kohlenstoffs schwankt zwischen 53-56 p. Ct. überschreitet aber niemals 57 p. Ct. 
Der Humus dient als direktes Nahrungsmittel für die Planze. Seine Absorption findet hauptsächlich in der Form von bumussaurem Ammoniak statt.

In den gewöhnlichen Erdarten entstelıt das humussaure Ammoniak hauptsächlich durch die Einwirkung des kohlensauren Ammoniaks auf den humussauren Kalk.

(Fortsetzung folgt.)

\title{
LXIV.
}

Ueber die Einwirkung des Chlors auf die Chlorverbindungen der Metalle bei Gegenwart von Chlormetallen der Alkalien.

\author{
Von
}

sobrero und selmi.

(Ann. de Cihm. et de Phys. XXIX. 161.)

Die Chlorverbindungen der Metalle, welche wir rorzugsweise zum Gegenstande unsrer Untersuçhung wählten, waren das Manganchlorür $\mathrm{MnCl}$ und das Bleichlorid $\mathrm{PbCl}$. Es wurden zwar noch einige Chlormetalle untersucht, die erhaltenen Resultate sind aber keineswegs vollkommen deutlich, so dass wir eine ausfübrliche Untersuchung derselben für spätere Zeit anfbewahren.

Chlor und Manganchlorür mit Chlormelallen der Alkalien.

Es ist bekannt, dass eine wässrige Lösung von Manganchlorïr durch. die Einwirkung von Chlorgas nicht verändert wird. Anders verhält es sich, wenn die Losung des Manganchlorür ein Chlormetall der Alkalien oder alkalischen Eiden (Chlorkalium, Chornatrium, Chlorcalcium u.s.w.) enthält. Das Chlor wirkt unter allen diesen Umständen sehr sclınell ein, denn kaum ist es in die Lösung gebracht worden, so zersetzt es das Manganoxydulsalz und erzeugt einen Niederschlag von Mangansuperoxyd. Der Versuch lüsst sich leicht auf die Weise anstellen, dass man einen Strom Chlorgas in eine Lüsung von vollkommen reinem Chlornatrium leitet, zu welcher man einige Tropfen reine Mangan-

Journ. f. prakt. Chemie L. 5. 